\title{
A THEOREM ON ALGEBRAS OF MEASURES ON TOPOLOGICAL GROUPS
}

\author{
by J. H. WILLIAMSON \\ (Received 2nd April 1958)
}

\section{Introduction}

Let $G$ be a locally compact Abelian group, and $\mathfrak{M}(G)$ the set of bounded complex (regular countably-additive Borel) measures on $G$. It is well known that $\mathfrak{M}(G)$ becomes a Banach space if the norm of $\lambda \in \mathfrak{M}(G)$ is defined by

$$
\|\lambda\|=\sup { }_{r}\left|\lambda\left(E_{r}\right)\right|
$$

the supremum being over all finite sets of disjoint Borel subsets of $G$. Let $C_{\infty}(G)$ be the Banach space of continuous complex functions on $G$, vanishing at infinity, with the uniform norm. It is known that $\mathfrak{M}(G)$ can be identified with the dual space of $C_{\infty}(G)$; every bounded linear functional is of the form

$$
\int_{G} f(x) d \lambda(x) \text { with } \lambda \in \mathfrak{M}(G),
$$

and conversely. We write $\lambda(f)$ for the value of this functional at $f$. The norm of $\lambda$ as a linear functional is the same as that defined above.

$\mathfrak{M}(G)$ becomes a Banach algebra if multiplication is defined by

$$
\lambda \star \mu(f)=\int_{G} \int_{G} f(x y) d \lambda(x) d \mu(y) .
$$

In this algebra there are certain obvious maximal ideals, the fundamental maximal ideals, of the form

$$
J_{x}=\left\{\lambda: \int_{\theta} \chi(x) d \lambda(x)=0\right\}
$$

where $\chi \in \hat{G}$ (the continuous characters on $G$ ). The measure $\lambda$ is determined by the values of

$$
\int_{G} \chi(x) d \lambda(x) \text { for } \chi \in \widehat{G}
$$

in general there exist maximal ideals which are not fundamental.

A Banach algebra is symmetric if there is, for each $\xi$, an element $\xi^{*}$ such that under every homomorphism into the complex field, $\xi$ and $\xi^{*}$ are mapped on to conjugate complex numbers. Writing

it is clear that

$$
\tilde{\lambda}(f)=\overline{\int_{G} f\left(x^{-1}\right) d \lambda(x)}
$$

$$
\tilde{\lambda}(E)=\overline{\lambda\left(E^{-1}\right)}
$$

for any Borel set $E$. Since evidently

$$
\int_{G} \chi(x) d \tilde{\lambda}(x)=\overline{\int_{G} \chi(x) d \lambda(x)}
$$

E.M.S. -N 
it follows that if $\mathfrak{M}(G)$ is symmetric, the element $\lambda^{*}$ corresponding to $\lambda$ must be $\tilde{\lambda}$ as defined above. If $\lambda=\tilde{\lambda}, \lambda$ will be called Hermitian.

Since the celebrated paper of Wiener and Pitt (3), it has been known that the algebra of measures on the real line has properties which are at first sight surprising. For instance, it is not symmetric (although Wiener and Pitt do not use this terminology, the fact is an easy corollary of their results). Sreider (2) gave a simpler proof on rather different lines. Recently, Hewitt (1) has extended Sreider's methods to the case of groups such that every neighbourhood of the identity contains an element of infinite order. In the present paper we adopt a somewhat different approach, more closely related to Wiener and Pitt's original method than to Sreider's, and are able to extend the results to all non-discrete groups. In particular we establish

Theorem 1. If $G$ is not discrete there exists on $G$ a Hermitian measure $\lambda$, such that, for any polynomial in $\lambda$,

$$
\left\|\sum_{r=0}^{n} a_{r} \lambda^{r}\right\|=\sum_{r=0}^{n}\left|a_{r}\right|
$$

From this, it can be shown that $\mathfrak{M}(G)$ is symmetric if and only if $G$ is discrete.

Since the details of the construction are rather complicated, it may be desirable to describe informally the basic ideas, which are simple. If the relation

$$
\left\|\Sigma a_{r} \lambda_{r}\right\|=\Sigma\left|a_{r}\right|
$$

is required, then the measures $\lambda_{r}$ must be, in a sense, independent. If their supports are disjoint, the required relation certainly holds. It also holds if the supports do not have too much in common; for instance if the measures have no atomic components, and the intersection of each pair of supports is countable. This is essentially the type of argument used by Sreìder and by Hewitt. The starting point of the present approach is the observation that we can allow the supports to have a great deal in common, and still have independence in the sense required. For instance (taking the case of two measures, for simplicity) it is possible to have

$$
\|a \lambda+b \mu\|=|a|+|b|
$$

for all $a, b$ even though the supports of $\lambda$ and $\mu$ are identical.

To see how this can happen, consider the measure

$$
\lambda=\underset{n=1}{\stackrel{\infty}{\star}} \lambda_{n}
$$

where $\lambda_{n}$ has mass $c(0<c<1)$ at $x_{n}$ and $1-c$ at $y_{n}$. The support consists of all points of the form $z=z_{1} z_{2} z_{3} \ldots$, where each $z_{n}$ is either $x_{n}$ or $y_{n}$. (We ignore all convergence problems here.) The use of a little elementary calculus leads to the conclusion that almost the whole of $\lambda$ is concentrated on points $z$ where the ratio of $x$ 's to $y$ 's in the sequence $z_{1}, z_{2}, z_{3}, \ldots$ is $\frac{c}{1-c}$. So if

$$
\mu=\underset{n=1}{\star} \mu_{n}
$$


where $\mu_{n}$ has mass $c^{\prime}$ at $x_{n}$ and $1-c^{\prime}$ at $y_{n}$, with $c^{\prime} \neq c$, then $\lambda$ and $\mu$ are concentrated on different subsets of their common support, and the required result follows. The extension to $n$ measures is immediate.

So what is required is to ensure that if $\lambda, \lambda^{2}, \lambda^{3}, \ldots$, have common supportor at least have supports which overlap substantially - then the "balance" is different for each power, so that each power is concentrated on a different part of the common support. The details of this vary according to the particular properties of the group under discussion.

It should be remarked that in many cases, for instance the real line, very considerable simplifications can be made, and a direct proof of Theorem $l$ is quite short. The complications of $\S 3$ of the present paper are largely due to the difficulties arising in groups where the identity has a neighbourhood consisting of elements of bounded order. It would have been rather simpler (although longer) to write out separate constructions in the three cases treated. It is hoped that the present treatment is a reasonable compromise between clarity and conciseness.

\section{Reduction to the Metrisable Case}

We show first that it is enough to consider groups in which the first axiom of countability is satisfied ; that is, where the identity (and hence each point) has a countable basic set of neighbourhoods. By a result of Kakutani, this is equivalent to the metrisability of the group.

Lemma 1. Let $x$ be a point in a locally compact topological space, and let $\left\{N_{\mathrm{a}}\right\}$ be a set of compact neighbourhoods of $x$, whose intersection is $x$. Then these neighbourhoods form a sub-basic set of neighbourhoods of $x$.

Proof. Let $N$ be any open neighbourhood of $x$. Then, for each $\alpha$, $P_{a}=N_{a} \backslash N$ is a compact set not containing $x$. Clearly

$$
\underset{a \in A}{\rightarrow a} P_{a}=\phi \text {, }
$$

and hence

$$
\bigcap_{n=1}^{k} P_{a}=\phi
$$

for some finite set of indices $a_{1}, a_{2}, \ldots, \alpha_{k}$. But then

$$
\bigcap_{n=1}^{k} N_{a_{n}} \subseteq N
$$

which is the required result.

Corollary. If $\left\{N_{n}\right\}$ is a decreasing countable set of neighbourhoods of $x$ whose intersection is $x$, it is basic.

Lemma 2. If $G$ is not discrete, there is a compact subgroup $H$ of $G$ such that $G / H$ is metrisable and not discrete.

Proof. Let $N_{1}$ be any compact symmetric neighbourhood of the identity 
element $e$ of $G$. For each $n>1$, let $N_{n}$ be a compact symmetric neighbourhood of $e$ such that $N_{n}^{2}$ is a proper subset of $N_{n-1}$. Consider

$$
H=\bigcap_{n=1}^{\infty} N_{n}
$$

it is easy to see that $H$ is a compact subgroup of $G$.

Let $\phi$ be the canonical map of $G$ on to $G / H$. Then, for each $n, \phi\left(N_{n}\right)$ is a neighbourhood of the identity $\bar{e}$ of $G / H$. Moreover, each $\phi\left(N_{n}\right)$ is compact, since $\phi$ is continuous, and

$$
\bigcap_{n=1}^{\infty} \phi\left(N_{n}\right)=\bar{e} .
$$

It follows, by the Corollary to Lemma 1, that these sets form a basic set of neighbourhoods of $\bar{e}$. That is, $G / H$ is metrisable.

Moreover, $G / H$ is not discrete. For each integer $n$ there is a point $x_{n}$ which is in $N_{n}$ but not in $N_{n+1}^{2}$. This implies that $\phi\left(x_{n}\right) \notin \phi\left(N_{n+1}\right)$; for if $\phi\left(x_{n}\right) \epsilon \phi\left(N_{n+1}\right)$ there would be a point $y \in H$ with $x_{n}=y z$, for $z \in N_{n+1}$. Since $H \subseteq N_{n+1}$, this would imply $y z \in N_{n+1}^{2}$, a contradiction. So $\phi\left(N_{1}\right)$ contains infinitely many distinct points $\phi\left(x_{1}\right), \phi\left(x_{2}\right), \phi\left(x_{3}\right), \ldots$. This implies that $G / H$ cannot be discrete; for $\phi\left(N_{1}\right)$ is compact, and in a discrete space a compact set is necessarily finite.

Lemma 3. Let $G$ be a locally compact group, $H$ a compact subgroup, with Haar measure on $H$ normalised to 1 . Then if $f(x) \in C_{\infty}(G)$, the function

$$
F(\bar{x})=\int_{H} f(x y) d y,
$$

where $x$ is any point in $\bar{x}$ and the integral is taken with respect to Haar measure on $H$, is in $C_{\infty}(G / H)$.

Proof. This is a routine verification.

Lemma 4. If the map $\psi$ is defined by

$$
F=\psi f,
$$

where $F$ and $f$ are as in Lemma 3 , then its dual map $\psi^{\prime}$ is an isometric $\sim-h o m o-$ morphism of $\mathfrak{M}(G / H)$ into $\mathfrak{M}(G)$.

Proof. Given $F \in C_{\infty}(G / H)$, there exists $f \in C_{\infty}(G)$ such that $F=\psi f$; it is only necessary to take $f(x)=F(\bar{x})$ for all $x \in \bar{x}$. Given $\Lambda \in \mathfrak{M}(G / H)$, and $\epsilon>0$, there is a function $F \in C_{\infty}(G / H)$ with $\|F\|=1$ such that

$$
|\Lambda(F)|>\|\Lambda\|-\epsilon \text {. }
$$

Let $f \in C_{\infty}(G)$ be as above ; then $\|f\|=1$ and

$$
\left|\left(\psi^{\prime} \Lambda\right)(f)\right|=|\Lambda(\psi f)|=|\Lambda(F)|>\|\Lambda\|-\epsilon,
$$

so that

$$
\left\|\psi^{\prime} \Lambda\right\|>\|\Lambda\|-\epsilon .
$$

But it is trivial that $\left\|\psi^{\prime}\right\|=1$, hence $\psi^{\prime}$ is an isometry. 
The fact that $\psi^{\prime}$ is a linear transformation is immediate. Also, for arbitrary $f \in C_{\infty}(G)$ and $\Lambda, M \in \mathfrak{M}(G / H)$,

$$
\begin{aligned}
\left(\psi^{\prime}(\Lambda \star M)\right)(f) & =\Lambda \star M(F) \\
& =\int_{G / H} \int_{G / H} \int_{H} f(x y u) d u d \Lambda(\bar{x}) d M(\bar{y}) \\
& =\int_{G / H} \int_{G / H} \int_{H} \int_{H} f(x y u v) d u d v d \Lambda(\bar{x}) d M(\bar{y}) \\
& =\int_{G / H} \int_{H} \int_{G} f(x y v) d \psi^{\prime} \Lambda(\bar{x}) d v d M(\bar{y}) \\
& =\int_{G} \int_{G} f(x y) d \psi^{\prime} \Lambda(\bar{x}) d \psi^{\prime} M(\bar{y}) \\
& =\left(\psi^{\prime} \Lambda \star \psi^{\prime} M\right)(f) .
\end{aligned}
$$

Hence

$$
\psi^{\prime}(\Lambda \star M)=\psi^{\prime} \Lambda \star \psi^{\prime} M
$$

Lastly, writing

$$
\tilde{f}(x)=\overline{f\left(x^{-1}\right)}
$$

and noting that

and

$$
\tilde{\lambda}(f)=\overline{\lambda(\tilde{f})}
$$

$$
\psi(\tilde{f})=\overline{\psi(\tilde{f})}
$$

it follows that

$$
\psi^{\prime}(\tilde{\Lambda})(f)=\overline{\left(\psi^{\prime} \Lambda\right)(\tilde{f})}=\overline{\Lambda(\psi \tilde{f})}=\overline{\Lambda(\psi \tilde{f})}=\tilde{\Lambda}(\psi f)=\psi^{\prime}(\tilde{\Lambda})(f)
$$

and hence

$$
\psi^{\prime}(\tilde{\Lambda})=\psi^{\prime}(\tilde{\Lambda})
$$

This completes the proof.

Corollary. If a measure satisfying the requirements of Theorem 1 can be constructed in each non-discrete metrisable group, such a measure can be constructed in each non-discrete group.

For, if $G$ is non-discrete and non-metrisable, let $H$ be a compact subgroup such that $G / H$ is non-discrete and metrisable. Then if $\Lambda$ is a measure on $G / H$ with the required properties, $\psi^{\prime}(\Lambda)$ is a measure on $G$ with the same properties.

\section{The Construction in a Metrisable Group}

In the following lemmas $m$ and $n$ denote positive integers.

Lemma 5. If

then $\rho_{m} \neq \rho_{n}$ if $m \neq n$.

$$
\rho_{n}=2^{-2 n} \frac{(2 n) !}{(n !)^{2}}
$$

Proof. $\rho_{n}=\prod_{r=1}^{n} \frac{2 r-1}{2 r} \neq \prod_{r=1}^{m} \frac{2 r-1}{2 r}=\rho_{m}$ 
Lemma 6. Let $p$ be an integer $\geqslant 3$, and let $v$ be the integral part of $\frac{n}{p}$. Then if $\sigma_{m} \neq \sigma_{n}$ if $m \neq n$.

$$
\sigma_{n}=2^{-2 n} \sum_{r=-\nu}^{\nu} \frac{(2 n) !}{(n-r p) !(n+r p) !}
$$

Proof. Write $\omega=\cos \frac{2 \pi}{p}+i \sin \frac{2 \pi}{p}$. Then

$$
\sigma_{n}=\frac{1}{p} \sum_{s=0}^{p-1}\left(\frac{\omega^{s}}{4}+\frac{1}{2}+\frac{\omega^{-s}}{4}\right)^{n}=\frac{1}{p} \sum_{s=0}^{p-1} \cos ^{2 n} \frac{s \pi}{p} .
$$

It follows that $\sigma_{m}<\sigma_{n}$ if $m>n$; for each term in the sum for $\sigma_{m}$ is less than the corresponding term in the sum for $\sigma_{n}$, with the exception of those corresponding to $s=0$, and $s=\frac{p}{2}$ (if present). (If $p=2$ then $\sigma_{n}=\frac{1}{2}$ for all $n$ ).

Lemma 7. If $a>0, b>0, a \neq b, a+b=1$, and $\nu$ is the integral part of $\frac{n}{2}$, and if

$$
\tau_{n}=\sum_{r=0}^{\nu} \frac{n !}{(2 r) !(n-2 r) !} a^{n-2 r} b^{2 r}
$$

then $\tau_{m} \neq \tau_{n}$ if $m \neq n$.

Proof. $\tau_{n}=\frac{1}{2}\left(1+(a-b)^{n}\right)$.

Lemma 8. Let $\left\{N_{n}\right\}$ be a basic set of compact neighbourhoods of e such that, for $n>1, N_{n}^{2} \subseteq N_{n-1}$. For each $n$, let $\mu_{n}$ be a positive measure of norm 1 whose support is contained in $N_{n}$. Then

$$
\stackrel{\infty}{\leftarrow}=1_{n=1}^{\infty} \mu_{n}
$$

converges to a positive measure $\mu$ of norm 1 whose support is contained in $N_{1}^{2}$, while for each positive integer $r$,

converges to $\mu^{r}$.

$$
\underset{n=1}{\infty} \mu_{n}^{r}
$$

The convolution-products converge both in the weak* topology of $\mathfrak{M}(G)$ and in the strong topology of operators on $L_{1}(G)$ (but not in general in the norm topology of $\mathfrak{M}(G)$ ).

Proof. Writing

and (if $m>n$ )

$$
\lambda_{n}=\mu_{1} \star \ldots \star \mu_{n},
$$

$$
\lambda_{m},{ }_{n}=\mu_{n+1} \star \ldots \star \mu_{m},
$$

it is easy to see that, for each $f \epsilon C_{\infty}(G)$ and $\epsilon>0$, there is an integer $m_{0}$ such that

$$
\left|f(x)-\left(\tilde{\lambda}_{m, n} \star f\right)(x)\right|<\epsilon,
$$

uniformly for $x \in G, m, n \geqslant m_{0}$. Since

$$
\tilde{\nu}_{1}\left(\nu_{2} \star f\right)=\left(\nu_{1} \star \nu_{2}\right)(f)
$$


it follows that

$$
\left|\lambda_{n}(f)-\lambda_{n}\left(\tilde{\lambda}_{m, n} \star f\right)\right|=\left|\lambda_{n}(f)-\lambda_{m}(f)\right|<\epsilon \text { for } m, n \geqslant m_{0} .
$$

Hence, as $n \rightarrow \infty, \lambda_{n}(f)$ tends to a limit; if this is denoted by $\mu(f)$, then it is immediate that $\mu$ is a bounded linear functional on $C_{\infty}(G)$, i.e., a measure. The other properties asserted are easily established.

In order to establish the strong convergence of $\lambda_{n}$ to $\mu$, let $g$ be a continuous function of compact support. Since

$$
\left\|\tilde{\lambda}_{m, n} \star g-g\right\| \rightarrow 0
$$

as $m, n \rightarrow \infty$, it follows that

$$
\left\|\lambda_{m} \star g-\lambda_{n} \star g\right\| \rightarrow 0
$$

as $m, n \rightarrow \infty$. Since continuous functions of compact support are dense in $L_{1}(G)$, the result follows.

Suppose now that for each $n, \mu_{n}$ is a positive measure of norm 1 ; and that $E_{n}, E_{n}^{\prime}$ are disjoint sets such that

$$
\mu_{n}\left(E_{n}\right)=a \text { and } \mu_{n}\left(E_{n}^{\prime}\right)=1-a
$$

where $0<\alpha<1$. Writing

$$
\lambda_{n}=\mu_{1} \star \ldots \star \mu_{n}
$$

it is clear that the support of $\lambda_{n}$ is the union of all sets of the form

$$
E=E^{(n)}=F_{1} F_{2} \ldots F_{n},
$$

where, for each $r, F_{r}$ is either $E_{r}$ or $E_{r}^{\prime}$. Let

$$
\begin{aligned}
w_{r}(E) & =1 \text { if } F_{r}=E_{r}, \\
& =0 \text { if } F_{r}=E_{r}^{\prime}, \\
W(E) & =\frac{1}{n} \sum_{r=1}^{n} w_{r}(E) .
\end{aligned}
$$

Lemma 9. Given $\epsilon>0, \eta>0$, there exists $m_{0}$ such that if $m \geqslant m_{0}$ and

$$
E_{\epsilon}^{(m)}=\bigcup E^{(m)},
$$

the union being over all $E^{(m)}$ such that

then

$$
\left|W\left(E^{(m)}\right)-a\right|<\epsilon,
$$

$$
\lambda_{m}\left(E^{(m)}\right)>1-\eta \text {. }
$$

Proof. If $t$ is a positive integer, and $E(t)$ is the union of all sets $E^{(m)}$ for which $W\left(E^{(m)}\right)=\frac{t}{m}$, then evidently

$$
\lambda_{m}(E(t))=\frac{m !}{t !(m-t) !} a^{t}(1-a)^{m-t} .
$$

Writing $t=s m$, this is asymptotically equal to

$$
(2 \pi m s(1-s))^{-\frac{1}{(}}\left(\frac{a^{s}}{s^{8}} \cdot \frac{(1-a)^{1-8}}{(1-s)^{1-8}}\right)^{m}
$$


for fixed $s$, as $m \rightarrow \infty$. Now the function

$$
f(s)=\left(\frac{a}{s}\right)^{s}\left(\frac{1-a}{1-s}\right)^{1-s}
$$

has a unique maximum at $s=\alpha$; at this maximum the function takes the value 1. Hence there is a positive real number $k$, less than 1 , such that

$$
f(s) \leqslant k \text { if }|s-a| \geqslant \epsilon .
$$

From this it follows that

$$
\left.\lambda_{m}\left(G \backslash E^{(m)}\right)=O_{(}^{(} m^{\frac{1}{t}} k^{m}\right) \rightarrow 0 \text { as } m \rightarrow \infty,
$$

which yields the desired conclusion.

Before proceeding to a proof of Theorem 1 , it is convenient to classify the groups considered as follows. Let $\mathfrak{t}$ be the set of integers $n$ such that every neighbourhood of $e$ contains an element of order $\geqslant n$. Write

We distinguish three cases :

$$
p=\sup _{n_{E} \text { in }} n
$$

$$
\begin{aligned}
& \text { (i) } p=\infty . \\
& \text { (ii) } 2<p<\infty . \\
& \text { (iii) } p=2 .
\end{aligned}
$$

In case (i), for every neighbourhood $N$ of $e$ and every integer $n$ there is in $N$ an element of order $\geqslant n$. In cases (ii) and (iii), for every neighbourhood $N$ of $e$ there is in $N$ an element of order $p$. For, in $N$ there is a neighbourhood $N^{\prime}$ which contains no element of order $>p$; and if there were in $N^{\prime}$ no element of order $p$ then $\sup n$ would be $<p$, a contradiction.

Since $G$ is non-discrete, the three cases listed above are the only possible ones.

If $x_{1}, \ldots, x_{n}$ are any points of $G$, write

$$
H\left(x_{1}, \ldots, x_{n}\right)=\cup \prod_{r=1}^{n} x_{r}^{a_{r}},
$$

where the union is over all sets of integers $\left\{a_{r}\right\}$ such that

$$
-n \leqslant a_{r} \leqslant n \text { for } 1 \leqslant r \leqslant n .
$$

The set $H$ is evidently finite.

In view of the Corollary to Lemma 4 , it is enough to prove Theorem 1 in the metrisable case. We treat Case (i), $p=\infty$, and indicate in parentheses the modifications required in the other cases (Case (ii), $2<p<\infty$; Case (iii), $p=2)$.

Proof of Theorem 1. Let $N_{1}^{\prime}, N_{2}^{\prime}, \ldots$ be a basic set of neighbourhoods of $e$. Let $N_{1}$ be a compact symmetric neighbourhood of $e$, contained in $N_{1}^{\prime}$, and let $x_{1} \in N_{1}$, Suppose that $N_{1}, \ldots, N_{n}$ and $x_{1}, \ldots, x_{n}$ have been chosen. 
Then choose $N_{n+1}$ to be a compact symmetric neighbourhood of $e$ such that

$$
\begin{gathered}
N_{n+1} \subseteq N_{n+1}^{\prime} \quad \ldots \ldots \\
N_{n+1}^{4 n+4} \cap H^{2}\left(x_{1}, \ldots, x_{n}\right)=\{e\} \\
N_{n+1}^{2} \subseteq N_{n} \quad \ldots \ldots \ldots
\end{gathered}
$$

and then choose $x_{n+1} \epsilon N_{n+1}$, of order $\geqslant n+1$ (order $p$; order 2).

For each $n$, let $\mu_{n}$ be the measure with mass $\frac{1}{2}$ at $e$, $\frac{1}{4}$ at $x_{n}, \frac{1}{4}$ at $x_{n}^{-1}\left(\frac{1}{2}\right.$ at $e$, $\frac{1}{4}$ at $x_{n}, \frac{1}{4}$ at $x_{n}^{-1} ; \frac{1}{3}$ at $e, \frac{2}{3}$ at $x_{n}$ ). In view of (1) and (3), by Lemma 8 the convolution-product

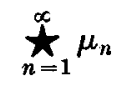

converges, say to $\mu$; and for each $m$ the convolution-product

converges to $\mu^{m}$.

$$
\underset{n=1}{\star} \mu_{n}^{m}
$$

Let $P(\mu)$ be a polynomial in $\mu$, of degree $h$; say

$$
P(\mu)=\sum_{r=0}^{h} a_{r} \mu^{r} .
$$

It is convenient to write, for $n \geqslant 1$,

$$
\nu_{n}=\mu_{n+h} .
$$

Then for $0 \leqslant r \leqslant h$, and each $n \geqslant 1$, we have

$$
\begin{aligned}
& \nu_{n}^{r}(\{e\})=2^{-2 r} \frac{(2 r) !}{(r !)^{2}}
\end{aligned}
$$

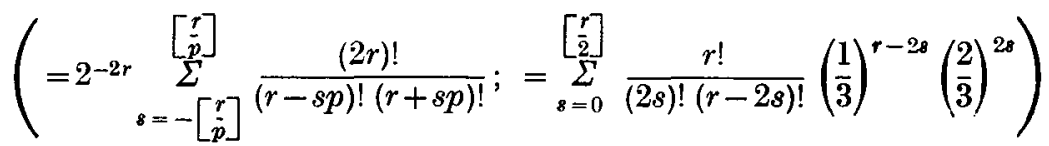

By Lemma 5 (Lemma 6 ; Lemma 7)

\section{Writing}

$$
v_{n}^{r}(\{e\}) \neq \nu_{n}^{R}(\{e\}) \text { if } r \neq R
$$

$$
\begin{aligned}
& E_{n}=\{e\} \\
& E_{n}^{\prime}=\left\{x_{n+h}^{-h}, \ldots, x_{n+h}^{-1}, x_{n+h}, \ldots, x_{n+h}^{h}\right\}
\end{aligned}
$$

for each $n$, we are in the situation of Lemma 9. Let

$$
\epsilon \leqslant \frac{1}{2} \min _{0<r<R<h}\left|v_{n}^{r}(\{e\})-v_{n}^{R}(\{e\})\right|
$$

and, using the notation of Lemma 9 , let $E_{r, \epsilon}^{(m)}$ be the union of all sets $E^{(m)}$ such that

$$
\left|W\left(E^{(m)}\right)-\nu_{n}^{r}(\{e\})\right|<\epsilon
$$


By Lemma 9, for given $\eta>0$ there exists $M$ such that

$$
\left(\underset{n=1}{\mathbb{M}} \nu_{n}^{r}\right)\left(E_{r, e}^{(M)}\right)>1-\eta
$$

for $0 \leqslant r \leqslant h, m \geqslant m_{1}$. Write

Then since

$$
E_{r, \epsilon}^{\prime}=H\left(x_{1}, \ldots, x_{h}\right) E_{r, \epsilon}^{\left(M_{1}\right)} N_{M_{1}+h+1}^{亡 h} .
$$

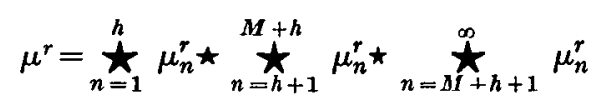

for $0 \leqslant r \leqslant h$, it is clear that for these values of $r$,

$$
\mu^{r}\left(E_{r, \epsilon}^{\prime}\right)>1-\eta
$$

Now, the sets $E_{r, \epsilon}^{\prime}$ are disjoint. For, suppose

where

$$
x_{1}^{a_{1}} \ldots x_{M+h}^{\alpha_{M}+h} y=x_{1}^{\beta_{1}} \ldots x_{M+h}^{\beta_{M+h}} z,
$$

$$
-h \leqslant \alpha_{i} \leqslant h, \quad-h \leqslant \beta_{i} \leqslant h
$$

and $y, z \in N_{M+h+1}^{2 h}$; then

$$
y^{-1} z=x_{1}^{a_{1}-\beta_{1}} \ldots x_{M+h}^{\mathrm{a}_{M}+h-\beta_{M+h}} \in N_{M+h+1}^{4 h} \subseteq N_{M+h+1}^{4(M+h+1)}
$$

Hence, by (2),

$$
x_{1}^{a_{1}-\beta_{1}} \ldots x_{M+h}^{\mathbf{a}_{M+h-\beta_{M+h}}}=e .
$$

But this implies $\alpha_{M+h}=\beta_{M+h}$, hence $\alpha_{M+h-1}=\beta_{M+h-1}, \ldots$, hence $\alpha_{h+1}=\beta_{h+1}$, by repeated use of (2). So, if $r \neq R$, the sets $E_{r, \epsilon}^{\prime}$ and $E_{R, \epsilon}^{\prime}$ are disjoint, since evidently $E_{r, \epsilon}^{(M)}, E_{R, \epsilon}^{(M)}$ are disjoint if $r \neq R$.

We then have

$$
\begin{aligned}
\| P(\mu) & \| \geqslant\left|P(\mu)\left(E_{0, \epsilon}^{\prime}\right)\right|+\ldots+P(\mu)\left(E_{h, \epsilon}^{\prime}\right) \mid \\
& \geqslant\left|a_{0}\right|(1-\eta)-\eta\left(\left|a_{1}\right|+\ldots+\left|a_{h}\right|\right)+\ldots+\left|a_{h}\right|(1-\eta)-\eta\left(\left|a_{0}\right|+\ldots+\left|a_{h-1}\right|\right) \\
& =\left(\left|a_{0}\right|+\left|a_{1}\right|+\ldots+\left|a_{h}\right|\right)(1-h \eta) .
\end{aligned}
$$

Since $\eta$ was arbitrary,

$$
\|P(\mu)\| \geqslant\left|a_{0}\right|+\left|a_{1}\right|+\ldots+\left|a_{h}\right| .
$$

But clearly the reverse inequality holds, hence

$$
\|P(\mu)\|=\left|a_{0}\right|+\left|a_{1}\right|+\ldots+\left|a_{h}\right|
$$

as required. This completes the proof.

\section{The Asymmetry of $\mathfrak{M}(G)$.}

Theorem 2. $\mathfrak{M}(G)$ is symmetric if and only if $G$ is discrete.

Proof. It is trivial that if $G$ is discrete then $\mathfrak{M}(G)$ is symmetric : for then $\mathfrak{M}(G)=L_{1}(G)$. 
If $G$ is not discrete, let $\mu$ be a measure satisfying the conditions of Theorem 1, and consider

$$
\nu=\frac{1}{2}\left(e-\mu^{2}\right),
$$

where now $e$ is the unit element of $\mathfrak{M}(G)$. Since

$$
\left\|\nu^{n}\right\|=2^{-n}\left(1+n+\frac{n(n-1)}{2 !}+\ldots\right)=1,
$$

the spectral radius of $\nu$ is 1 ; there is thus a multiplicative linear functional $m^{\prime}$ such that

which implies

$$
\left|m^{\prime}(\nu)\right|=1
$$

and hence

$$
m^{\prime}\left(\mu^{2}\right)=-1
$$

In either case

$$
m^{\prime}(\mu)= \pm i
$$

$$
m^{\prime}(\tilde{\mu})=m^{\prime}(\mu) \neq \overline{m^{\prime}(\mu)}
$$

so that $\mathfrak{M}(G)$ is not symmetric.

Remark. If $\mu$ is as above, and $m^{\prime}(\mu)=i$, and if $\lambda=\frac{1}{2}(e-i \mu)$, then $m^{\prime}(\lambda)=1$ whereas $m^{\prime}(\tilde{\lambda})=0$.

Theorem 3. The fundamental maximal ideals constitute the \$ilov boundary of the maximal ideals of $\mathfrak{M}(G)$ if and only if $G$ is discrete.

Proof. If $G$ is discrete then $\mathfrak{M}(G)=L_{1}(G)$ and so every maximal ideal of $\mathfrak{M}(G)$ is fundamental.

If $G$ is not discrete, let $\mu, \nu$ and $m^{\prime}$ be as in Theorem 2. Then, since $\mu$ is Hermitian, $m(\mu)$ is real for each fundamental homomorphism $m$, hence

$$
0 \leqslant m(\nu) \leqslant \frac{1}{2}
$$

for all such $m$. Since $\left|m^{\prime}(\nu)\right|=1$, the conclusion is immediate.

Remark. It may be worth while to point out why $m(\mu)$ is real for each fundamental homomorphism $m$, whereas it is not real for all homomorphisms. It is easily seen that if $m$ is any homomorphism, $m\left(\mu_{n}\right)$ is real for each $n$, where $\mu_{n}$ is as in the proof of Theorem 1. But it does not follow in general that $m(\mu)$ is real; since the convolution-product is not convergent to $\mu$ in the norm topology of $\mathfrak{M}(G)$, it is not in general true that

$$
m(\mu)=\lim _{p \rightarrow \infty} \prod_{n=1}^{p} m\left(\mu_{n}\right) .
$$

However, if there exists $f \in L_{1}(G)$ such that $m(f) \neq 0$, which is so if and only if $m$ is fundamental, then

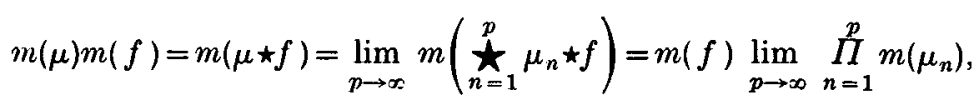

by Lemma 8 , and the desired conclusion follows in this case. 
Theorem 4. $|m(\lambda)| \geqslant c>0$ for each fundamental homomorphism $m$ implies the existence of $\lambda^{-1}$ if and only if $G$ is discrete.

Proof. If $G$ is not discrete, and $\mu, m^{\prime}$ are as in Theorem 2, then clearly

$$
m^{\prime}\left(e+\mu^{2}\right)=0,
$$

so that $\left(e+\mu^{2}\right)$ has no inverse, whereas

$$
m\left(e+\mu^{2}\right) \geqslant 1
$$

for each fundamental homomorphism $m$.

If $G$ is discrete, every homomorphism is fundamental.

\section{Acknowledgments}

The writer wishes to place on record his gratitude to Professor Edwin Hewitt, and to Dr I. T. Adamson, for advice and criticism.

Added in proof: A much less complicated proof of Theorem 1 has been obtained by W. Rudin (Bull. Amer. Math. Soc., 65 (1959), 227-247).

\section{REFERENCES}

(1) E. Hewitt, The asymmetry of certain algebras of Fourier-Stieltjes transforms, Michigan Math. J., 5 (1958), 149.158.

(2) $\mathrm{Yu}$. A. Sreĩder, The structure of maximal ideals in rings of measures with convolution, Mat. Sbornik N.S., 27 (69) (1950), 297-318; American Mathematical Society Translation no. 81 .

(3) N. Wiener and H. R. Pitt, On absolutely convergent Fourier-Stieltjes transforms, Duke Math. J., 4 (1938), 420-436.

QUEEN'S UNTVERSTTY

BeLFAST 